\title{
CONJUGACY SEPARABILITY OF THE GROUPS OF HOSE KNOTS
}

\author{
BY \\ PETER F. STEBE
}

\begin{abstract}
Let $G$ be a group. An element $g$ of $G$ is c.d. in $G$ if and only if, given any element $h$ of $G$, either $h$ is conjugate to $g$ or there is a homomorphism $\xi$ from $G$ onto a finite group such that $\xi(g)$ is not conjugate to $\xi(h)$. Following A. Mostowski, a group is conjugacy separable or c.s. if and only if every element of the group is c.d. In this paper we show that the groups of hose knots are c.s.
\end{abstract}

According to K. Brauner [1], the groups of hose knots are presented as follows: The groups have generators $P_{1}, Q_{1}, \ldots, Q_{r}$ and relations

$$
Q_{1}^{-m_{i}} P_{1}^{n_{1}}=1, \quad Q_{i}^{m_{i}} P_{i}^{n_{i}-n_{i-1} m_{i}} Q_{i-1}^{m_{i}{ }^{m_{i}}}=1, \quad m_{i} k_{i}=n_{i} u_{i}+1,
$$

where

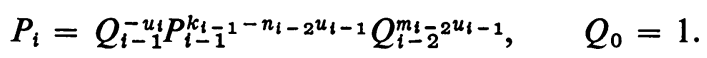

Clearly these groups can be obtained from a free cyclic group by repeated addition of roots. An element $u$ of a group $G$ is conjugacy distinguished or c.d., in $G$, if for every element $v$ of $G$ not conjugate to $u$ there is a homomorphism $\xi$ from $G$ onto a finite group such that $\xi(u)$ and $\xi(v)$ are not conjugate. Following A. Mostowski [5], a group is c.s., or conjugacy separable, if every element is c.d. Mostowski proves that the conjugacy problem can be solved in c.s. groups. In this paper we will show that the groups of hose knots are c.s.

A general reference for theorems in infinite group theory is the book by $\mathrm{W}$. Magnus, A. Karass and D. Solitar [4].

A group $G$ is $\Pi_{c}$ if and only if given any two elements $a$ and $b$ of $G$ either $a=b^{z}$ for an integer $z$ or there is a normal subgroup $N$ of finite index in $G$ such that $a \not \equiv b^{z} \bmod N$ for all $z$. Let $G$ be a $\Pi_{c}$ group. Let $H=\left(x, G ; x^{m}=g\right)$ for $m>1$ and $g \in G$ be the free product of $G$ and a cyclic group generated by $x$ with a cyclic amalgamated subgroup generated by $g$. According to [6, Corollary 3.3], $H$ is $\Pi_{c}$. It is also shown in [6] that free groups and groups with a free subgroup of finite index are $\Pi_{c}$. Since the intersection of finitely many subgroups of finite index in a group $G$ is again of finite index in $G$, it follows that if $t_{1}, \ldots, t_{n}$ and $b$ are elements of $G$ such that $t_{i} \neq b^{z_{i}}$ for all $z_{i}$ and all $i=1, \ldots, n$, and if $G$ is $\Pi_{c}$, then there is a

Received by the editors May 4, 1970.

AMS 1970 subject classifications. Primary 20E25, 20E40, 55A25.

Key words and phrases. Group, conjugacy, separable group, conjugacy problem, knot group.

Copyright (C) 1971, American Mathematical Society 
single normal subgroup $N$ of finite index in $G$ such that no congruence $t_{i} \equiv b^{z_{i}} \bmod N$ has an integral solution.

LEMMA 1. Let $F$ be a free group. Let $\alpha_{i}$ be automorphisms of $F$ and let $b_{i}=\alpha_{i}(c)$ for an element $c$ of $F$. Suppose the equations $a_{1}=b_{1}^{-n_{0}} c^{n_{1}}, \ldots, a_{i}=b_{i}^{-n_{i-1}} c^{n_{i}}, \ldots, a_{m}=$ $b_{m}^{-n_{m-1}} c^{n_{0}}$ have no integral solution $n_{0}, \ldots, n_{m-1}$. There is a normal subgroup $N$ of finite index in $F$ such that the system of equations has no solution modulo $N$.

Proof. If $c$ is the identity element, all $b_{i}$ are the identity. In this case the existence of $N$ follows from the fact that $F$ is residually finite. Thus we can assume that $c$ is not the identity.

Suppose there is a subscript $i$ such that $b_{i}$ commutes with $c$. By the symmetry of the equations we can assume $\left(b_{1}, c\right)=1$. Since $F$ is free, there is an element $u$ of $F$ such that $b_{1}=u^{r}, c=u^{s}$. Since $\alpha_{1}(c)=b_{1}, \alpha_{1}\left(u^{r}\right)=u^{s}$. It follows from an easy extension of Theorem 7 of [6] that $r=+s$ or $r=-s$, so that $b_{1}=c$ or $b_{1}=c^{-1}$. If $b_{1}=c$, the given set of equations is equivalent to $a_{1}=c^{n_{1}-n_{0}}, a_{2}=b_{2}^{-n_{1}} c^{n_{2}}, \ldots, a_{m} a_{1}$ $=b_{m}^{-n_{m-1}} c^{n_{1}}$. If $b_{1}=c^{-1}$, the system of equations is equivalent to $a_{1}=c^{n_{0}+n_{1}}, a_{2}$ $=\left(b_{2}^{-1}\right)^{n_{1}} c^{n_{2}}, \ldots, a_{m} a_{1}^{-1}=b_{m}^{-n_{m-1}} c^{n_{1}}$. Since $b_{2}=\alpha_{2}(c), b_{2}^{-1}=\alpha_{2}\left(c^{-1}\right)$ so that $b_{2}^{-1}=$ $\alpha_{2}\left(\alpha_{1}(c)\right)$. Thus for $b_{1}=c$ or $c^{-1}$ the original set of equations is equivalent to a set of equations of the same form with fewer equations and a single equation $a_{1}=c^{z}$. If the equation $a_{1}=c^{z}$ has no solution, the fact that $F$ is $\Pi_{c}$ implies that there is a normal subgroup $N$ of finite index in $F$ such that $a_{1} \neq c^{z} \bmod N$ for all $z$. But the original set of equations will have no solution modulo $N$. Thus we can ignore the equation $a_{1}=c^{z}$. Repeating this process, it is clear that we can reduce the original set of equations to a set of equations of the same form with $\left(b_{i}, c\right) \neq 1$ for all $i$. We assume that $\left(b_{i}, c\right) \neq 1$.

Suppose there is a subscript $i$ such that $a_{i}=b_{i}^{x} c^{y}$ has no solution for integers $x$ and $y$. According to Lemma 3 of [8], there is a normal-subgroup $N$ of finite index in $F$ such that $a_{i}=b_{i}^{x} c^{y}$ has no solution modulo $N$. Clearly the set of simultaneous equations has no solution modulo $N$. Now suppose that for each $i$ there are integers $h_{i}$ and $k_{i}$ such that $a_{i}=b_{i}^{-k_{i}} c^{h_{i}}$. Since the set of equations has no solution, at least one difference $h_{i}-k_{i+1}, h_{m}-k_{0}$, is not zero. Let $p$ be a prime relatively prime to at least one nonzero difference. Since $\left(b_{i}, c\right) \neq 1$ for all $i$, it follows from a theorem of M. Hall [2] that there is a normal subgroup $N$ of finite indēx in $F$ such that $F / N$ is a $p$-group and $\left(b_{i}, c\right) \notin N$ for all $i$. Suppose, to obtain a contradiction, that the given set of equations has a solution modulo $N$. There are integers $v_{0}, \ldots, v_{m-1}$ such that $a_{i} \equiv b_{i}^{-v_{i-1}} c^{v_{i}} \bmod N\left(v_{m}=v_{0}\right)$. Since $a_{i}=b_{i}^{-k_{i}} c^{h_{i}}$ we have $b_{i}^{v_{i-1}-k_{i}} \equiv c^{v_{i}-h_{i}} \bmod N$. Since $\left(b_{i}, c\right) \notin N$, neither $v_{i-1}-k_{i}$ nor $v_{i}-h_{i}$ can be relatively prime to $p$. Thus $p$ divides each difference $h_{i}-k_{i+1}$. But this result contradicts the definition of $p$. Thus the system of equations has no solution modulo $N$.

Let us define a property $\Pi$ of an element of a group $G$ as follows. Let $G$ be a group. An element $g$ of $G$ has property $\Pi$ if given a set of equations $a_{1}=$ 
$g^{-r_{0}} b_{1} g^{r_{1}}, \ldots, a_{i}=g^{-r_{i-1}} b_{i} g^{r_{i}}, \ldots, a_{m}=g^{-r_{m-1}} b_{m} g^{r_{0}}$ for $a_{i}$ and $b_{i}$ elements of $G$ then either there are integers $r_{0}, \ldots, r_{n-1}$ such that the equations are true or there is a normal subgroup $N$ of finite index in $G$ such that the system of equations has no solution modulo $N$.

LEMMA 2. If $G$ is a group with a free normal subgroup of finite index, every element of $G$ has property $\Pi$.

Proof. Let $F$ be a free normal subgroup of finite index in $G$. Suppose the equations $a_{i}=c^{-r_{i-1}} b_{i} c^{r_{i}}, r_{m}=r_{0}$, have no solution. If these equations have no solution modulo $F$, then $F$ is the subgroup required by the lemma. Assume that there is a finite set of solutions $r_{0, k}, \ldots, r_{m-1, k}$ to the equations modulo $F$, and that for distinct $k$ the solutions are incongruent modulo the order of $c$ modulo $F$. Let $c$ have order $h$ modulo $F$. Now the equations $a_{i}=c^{-r_{i-1, k}-t_{i-1} h} b_{i} c^{r_{i, k}+t_{i} h}$ have no solution $t_{0}, \ldots, t_{m-1}$ with $t_{m}=t_{0}$ and these latter equations are equivalent to the system of equations $b_{i}^{-1} c^{r_{i-1, k}} a_{i} c^{-r_{i, k}}=\left(b_{i}^{-1} c^{h} b_{i}\right)^{-t_{i-1}}\left(c^{h}\right)^{t_{i}}$. Since these equations are in $F$, it follows from Lemma 1 that there is a single normal subgroup $N$ of finite index in $F$ such that none of these equations has a solution modulo $N$. Let $M$ be the intersection of all the conjugates of $N$ in $G$. Since $F$ is of finite index in $G, M$ is of finite index in $G$. Clearly, the original system of equations has no solution modulo $M$.

LEMMA 3. Let $A_{1}$ be a free cyclic group. If every element of $A_{2}$ has property $\Pi$, then every element of $A_{1} \times A_{2}$ has property $\Pi$.

Proof. Let $G$ be the direct product of $A_{1}$ and $A_{2}$. Let $a_{i}, b_{i}$ for $i=1, \ldots, m$ and $c$ be elements of $G$. Suppose that the equations $a_{i}=c^{-r_{i-1}} b_{i} c^{r_{i}}, r_{0}=r_{m}$, have no solution. Suppose the equations have no solution modulo $A_{2}$. Since every element of $A_{1}$ has property $\Pi$, there is a normal subgroup $N$ of finite index in $A_{1}$ such that the system of equations has no solution modulo $A_{2} \times N$. Hence we assume that the system of equations has a solution modulo $A_{2}$. Let $a_{i}=a_{i, 1} a_{i, 2}, b_{i}=b_{i, 1} b_{i, 2}$ and $c=c_{1} c_{2}$ where $a_{i, j}, b_{i, j}$ and $c_{j}$ are elements of $A_{j}$. By our assumption, there are integers $s_{0}, \ldots, s_{m-1}$ such that $a_{i, 1}=c_{1}^{-s_{i-1}} b_{i, 1} c_{1}^{s_{i}}$ and $s_{m}=s_{0}$. If the equations $a_{i, 2}=c_{2}^{-u} c_{2}^{-s_{i}-1} b_{i, 2} c_{2}^{s_{i}} c_{2}^{u}$ have a solution $u$, it follows from the fact that $A_{1}$ is abelian that the original set of equations has a solution $s_{i}+u$. Thus the latter equations have no solution $u$. It will be shown below that there exists a normal subgroup $N_{2}$ of $A_{2}$ such that the latter set of equations has no solution modulo $N_{2}$. Since $A_{2}$ is residually finite, it is clear that $N_{2}$ can be chosen so that $c_{2}$ is not an element of $N_{2}$. Let $h$ be the order of $c_{2}$ modulo $N_{2}$. Let $N_{1}$ be a normal subgroup of finite index in $A_{1}$ such that $c_{1}$ has order $h$ modulo $N_{1}$. Since $A_{1}$ is free cyclic, $N_{1}$ is the subgroup generated by $c^{h}$. Let $K=N_{1} \times N_{2}$.

Suppose $a_{i}=c^{-r_{i-1}} b_{i} c^{r_{i}}, r_{0}=r_{n}$, has a solution $r_{0}, \ldots, r_{m-1}$ modulo $K$. We have $b_{i, 1}^{-1} a_{i, 1} \equiv c_{1}^{r_{i}-r_{i-1}} \bmod K$. Since $b_{i, 1}^{-1} a_{i, 1}=c^{s_{i}-s_{i-1}}, r_{i}-r_{i-1} \equiv s_{i}-s_{i-1} \bmod h$. Here subscripts on $r$ and $s$ are taken modulo $m$. Summing these congruences we obtain $r_{i}-r_{0} \equiv s_{i}-s_{0} \bmod h$ so that $r_{i}=s_{i}+\left(r_{0}-s_{0}\right)+q_{i} h$. Since $h$ is the order of $c_{2}$ modulo 
$N_{2}$ we have $a_{i, 2} \equiv c_{2}^{-u} c_{2}^{-s_{i}-1} b_{i, 2} c_{2}^{s_{i}} c_{2}^{u} \bmod N_{2}$ for $u=r_{0}-s_{0}$. Since this contradicts the definition of $N_{2}$, the lemma is proven.

Now we will show that if a set of equations $d_{i}=g^{-u} e_{i} g^{u}$ for $i=1, \ldots, n$ has no solution $u$ for $g, e_{i}$, and $d_{i}$ in $A_{2}$ then there is a normal subgroup $N_{2}$ of finite index in $A_{2}$ such that these equations have no solution modulo $N_{2}$. Note that since every element of $A_{2}$ has property $\Pi$, given elements $a$ and $b$ of $A_{2}$ either the equations $a=b^{-r_{0}} b b^{r_{1}}, a^{-1}=b^{-r_{1} b^{-1}} b^{r_{0}}$ have a solution $r_{0}, r_{1}$ or there is a normal subgroup $N$ of finite index in $A_{2}$ such that these equations have no solution modulo $N$. Thus $A_{2}$ is $\Pi_{c}$.

Suppose that there is a subscript $i$ such that $e_{i}^{-1} g^{r} e_{i}=g^{s}$ implies $g^{r}=g^{s}=1$. By the symmetry of the equations we can assume that this subscript is 1 . Consider the $n$ systems of equations:

$$
\begin{aligned}
d_{1} & =g^{-r_{0}} e_{1} g^{r_{1}}, & g & =g^{-r_{1}} g g^{r_{0}}, \\
d_{1} & =g^{-r_{0, i}} e_{1} g^{r_{1, i}}, & d_{1}^{-1} & =g^{-r_{1, i}} e_{1}^{-1} g^{r_{2, i}}, \\
d_{i} & =g^{-r_{2, i}} e_{i} g^{r_{0, i}}, & i & =2, \ldots, n .
\end{aligned}
$$

Suppose each of these systems has a solution. The first set of equations implies that $d_{1}=g^{-r_{0}} e_{1} g^{r_{0}}$. If $n$ is greater than one, the other sets of equations imply that $d_{i}=g^{-r_{0, i} e_{i}} g^{r_{0, i}}$ and $d_{1}=g^{-r_{0, i}} e_{1} g^{r_{1, i}}$. Thus $g^{-r_{0}} e_{1} g^{r_{0}}=g^{-r_{0, i}} e_{1} g^{r_{1, i}}$. It follows from the assumption that $g^{r_{0, i}}=g^{r_{0}}$. Thus if the above systems of equations have solutions, there is a solution to $d_{i}=g^{-u} e_{i} g^{u}, i=1, \ldots, n$. Thus at least one of these systems has no solution. Since $g$ has property $\Pi$, there is a normal subgroup $N$ of finite index in $A_{2}$ such that at least one of these equations has no solution modulo $N$. If the system of equations $d_{i}=g^{-u} e_{i} g^{u}, i=1, \ldots, n$, has a solution modulo $N$, clearly every one of the other systems of equations has a solution modulo $N$.

Now suppose that for each $i$ there are integers $r_{i}$ and $s_{i}$ such that $e_{i}^{-1} g^{r_{i}} e_{i}=g^{s_{i}}$. Let $r$ be the least common multiple of the $r_{i}, i=1, \ldots, n$. For each $i$ there is an integer $v_{i}$ such that $e_{i}^{-1} g^{r} e_{i}=g^{v_{i}}$. Since $A_{2}$ is $\Pi_{c}$, it follows from Theorem 7 of [6], that either $g$ has finite order or each $v_{i}$ is $r$ or $-r$. We now consider several cases.

Suppose that $g$ has finite order or, for each $i, v_{i}=r$. In this case there are but finitely many distinct $n$-tuples of the form $\left(d_{1}^{-1} g^{-u} e_{1} g^{u}, \ldots, d_{n}^{-1} g^{-u} e_{n} g^{u}\right)$. Since at least one entry in each $n$-tuple is not the identity and $A_{2}$ is residually finite, there is a single normal subgroup $N_{2}$ of finite index in $A_{2}$ such that at least one entry in each of the $n$-tuples is not an element of $N_{2}$. Thus the equations $d_{\imath}=g^{-u} e_{i} g^{u}$ have no solution modulo $N_{2}$.

Suppose that $g$ has infinite order and that, for at least one subscript $i, v_{i}=-r$. We can rearrange the equations so that $v_{1}=\cdots=v_{k}=-r, v_{k+1}=\cdots=v_{n}=r$. Let $\bar{d}_{1}=d_{1}, \bar{d}_{i}=d_{1} d_{i}$ for $1<i \leqq k$, and $\bar{d}_{i}=d_{i}$ for $i>k$. The system of equations $d_{i}=$ $g^{-u} e_{i} g^{u}$ has a solution $u$ if and only if $u$ solves the system $\bar{d}_{i}=g^{-u} \bar{e}_{i} g^{u}$ where $\bar{e}_{1}=e_{1}, \bar{e}_{i}=e_{1} e_{i}$ for $1<i \leqq k$ and $\bar{e}_{i}=e_{i}$ for $i>k$. For all $i>1$ we have that $\bar{e}_{i}^{-1} g^{r} \bar{e}_{i}=g^{r}$. Also $\bar{e}_{1}^{-1} g^{r} \bar{e}_{1}=g^{-r}$. If there is an integer $t$ such that $0 \leqq t<r$ and the 
equations $g^{-t} \bar{e}_{1}^{-1} g^{t} \bar{d}_{1}=\left(g^{2 r}\right)^{z}, \quad \bar{d}_{2}=g^{-t} \bar{e}_{2} g^{t}, \ldots, \bar{d}_{n}=g^{-t} \bar{e}_{n} g^{t}$ have an integral solution $z$, then for $u=t+r z$ we have that $\bar{d}_{i}=g^{-u} \bar{e}_{i} g^{u}$. Thus for all $t$ between zero and $r$ the latter system has no solution. Since $A_{2}$ is $\Pi_{c}$, there is a single normal subgroup $N_{2}$ of finite index in $A_{2}$ such that for all $t$ between zero and $r$, the latter system of equations has no solution. Clearly, $d_{i}=g^{-u} e_{i} g^{u}$ has no solution modulo $N_{2}$.

LEMMA 4. Let $G$ be the free product of groups $A$ and $B$ with a cyclic amalgamated subgroup generated by an element $h$ of $G$. Let $G / G^{\prime}$ be free cyclic and let $h \notin G^{\prime}$. If $u$ is a cyclically reduced element of length greater than one in $G$, and $u^{2} b u^{-n}$ for $n>0$ begins with a syllable in a different factor from the first syllable of $u$, then either $b$ commutes with $u$ or $u^{-1}$ is an initial segment of $b$.

This lemma was suggested by Lemma 1 in S. Lipschutz [3].

Proof. Suppose $u^{-1}$ is not an initial segment of $u$, i.e., if $b=b_{1} \cdots b_{j}$ in reduced form, we do not have $b_{1} \cdots b_{k}=u^{-1} h^{e}$ where $h^{e}$ is in the amalgamated subgroup. Let $u=a_{1} \cdots a_{m}$ be $u$ written in reduced form. Since $u$ is cyclically reduced, we have not only adjacent $a_{i}$ in different factors of $G$ but also $a_{1}$ and $a_{m}$ are not in the same factor of $G$. Thus $u^{2} b u=a_{1} \cdots a_{m} a_{1} \cdots a_{m} b_{1} \cdots b_{j} u^{-n}$. If the bold syllable $a_{m}$ cancels, it cannot be by an element of $b$, since $u^{-1}$ is not an initial segment of $b$. Thus we have $u^{2} b u^{-n}=a_{1} \cdots a_{m} h^{k} a_{i}^{-1} \cdots a_{1}^{-1} u^{-p}$, so that $h^{k_{1}} a_{i}^{-1}$ cancels with the bold $a_{m}$, and $h^{k_{1}}$ is in the amalgamated subgroup. If cancellation reaches the initial syllable $a_{1}$, we have $a_{1} \cdots a_{m} h^{k_{1}} a_{i}^{-1} \cdots a_{1}^{-1} a_{m}^{-1} \cdots a_{i+1}^{-1}=h^{k_{2}}$. Since $u^{2} b u^{-n}=$ $a_{1} \cdots a_{m} h^{k_{1}} a_{i}^{-1} \cdots a_{m}^{-1} u^{-p}$ we have $b=u^{-1} h^{k_{1}} a_{i+1} \cdots a_{m} u^{r}$. Since $G / G^{\prime}$ is free cyclic

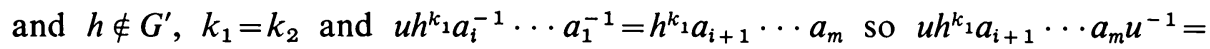
$h^{k_{1}} a_{i+1} \cdots a_{m}$. This implies that $u$ commutes with $b$.

LEMMA 5. Let $G$ and $u$ satisfy the hypotheses of Lemma 4. If $b$ does not commute with $u$, there is an integer $n_{0}$ such that if $n=n_{0}$ or $-n_{0}$ and $m=n /|n|$, then the first syllable of $u^{-n} b u^{n}$ is in the same factor of $G$ as the first syllable of $u^{-m}$, and the last syllable of $u^{-n} b u^{n}$ is in the same factor of $G$ as the last syllable of $u^{m}$.

Proof. If $u$ or $u^{-1}$ is an initial segment of $b$, it follows from the fact that $u$ has finite syllable length that there is an integer $r$ such that $b=u^{r} b^{\prime}$ where $u$ and $u^{-1}$ are not initial segments of $b^{\prime}$. If $u$ or $u^{-1}$ is a terminal segment of $b^{\prime}$, there is an integer $s$ such that $b^{\prime}=\boldsymbol{b} u^{s}$ and neither $u$ nor $u^{-1}$ is a terminal segment of $b$. Now if $r$ or $s$ is not zero, the syllable length of $b$ is greater than the syllable length of $b$. If the above process is repeated we must eventually obtain integers $x$ and $y$ and a group element $g$ such that $b=u^{x} g u^{y}$, where neither $u$ nor $u^{-1}$ is an initial or terminal segment of $g$. Since $b$ does not commute with $u, g$ does not commute with $u$. Let

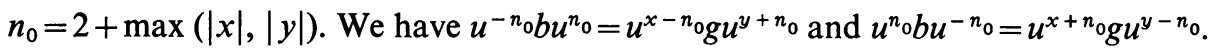
In each expression the exponents on $u$ have opposite signs and each exponent in an expression involving $g$ is greater than or equal to 2 in absolute value. The lemma now follows from Lemma 4. 
Let $G$ be a group, $n$ an integer greater than 1, and let $g$ be an element of $g$. Let $H$ be the free product of $G$ and a cyclic group generated by $x$ with the subgroups generated by $x^{n}$ and $g$ amalgamated. We write $H=\left(x, G ; x^{n}=g\right)$.

LemMa 6. Let $G$ be a $\Pi_{c}$ group. Let $H=\left(x, G ; x^{m}=g\right)$ for $g \in G, g \notin G^{\prime}$ and $m>1$. If $G / G^{\prime}$ and $H / H^{\prime}$ are free cyclic, $u$ is a cyclically reduced element of length greater than one in $H$ and $u \notin H^{\prime}$, then $u$ has property $\Pi$ in $H$.

Proof. Suppose the equations $a_{i}=u^{-r_{i-1}} b_{i} u^{r_{i}}, i=1, \ldots, n, r_{0}=r_{n}$, have no solution, where $a_{i}$ and $b_{i}$ are elements of $H$. We consider several cases.

Suppose every $b_{i}$ commutes with $u$. In this case the given system of equations is equivalent to the system $a_{i} b_{i}^{-1}=u^{r_{i}-r_{i-1}}$ for $i=1, \ldots, n-1$ and $a_{1} \cdots a_{n}=b_{1} \cdots b_{n}$. Since the $r_{i}-r_{i-1}$ are independent, the first $n-1$ of these equations can be replaced by $a_{i}=u^{z_{i}}$ for $z_{i}$ an arbitrary integer. Thus at least one of the equations in the latter set has no solution. Since Corollary 3.3 [6] implies that $H$ is $\Pi_{c}$ and hence residually finite, there is a normal subgroup $N$ of finite index in $H$ such that at least one of the equations of the latter set has no solution modulo $N$. But then the original system of equations has no solution modulo $N$.

Now suppose that at least one of the $b_{i}$ does not commute with $u$. By the symmetry of the set of equations we can assume that $b_{1}$ does not commute with $u$. Let $\eta$ be the natural homomorphism from $H$ to $H / H^{\prime}$ and let $v$ be a generator of the free cyclic group $H / H^{\prime}$. Let $\eta\left(a_{1}\right)=v^{e_{1}}, \eta\left(b_{1}\right)=v^{e_{2}}$ and $\eta(u)=v^{\text {s. Now }} \eta\left(a_{1}\right)=$ $\eta(u)^{-r_{0}} \eta\left(b_{1}\right) \eta(u)^{r_{1}}$ implies that $e_{1}=e_{2}+s\left(r_{1}-r_{0}\right)$. We consider several cases.

Suppose that $s$ does not divide $e_{1}-e_{2}$. Then the equations

$$
\eta\left(a_{i}\right)=\eta(u)^{-r_{i-1}} \eta\left(b_{i}\right) \eta(u)^{r_{i}}, \quad i=1, \ldots, n, \quad r_{0}=r_{n},
$$

have no solution. Since $H / H^{\prime}$ is free cyclic, $\eta(u)$ has property $\Pi$ in $H / H^{\prime}$ and there is a homomorphism $\xi$ from $H / H^{\prime}$ onto a finite group so that

$$
\xi \eta\left(a_{i}\right)=\xi \eta(u)^{-u_{r-1}} \xi \eta\left(b_{i}\right) \xi \eta(u)^{r_{i}}, \quad i=1, \ldots, n, \quad r_{0}=r_{n},
$$

has no solution. If $K$ is the kernel of $\xi \eta$, the given set of equations has no solution modulo $K$ and $K$ is a normal subgroup of finite index in $H$.

If $s$ divides $e_{1}-e_{2}$, set $k=\left(e_{1}-e_{2}\right) / s$. Consider the equation $a_{1}=u^{-r_{0}}\left(b_{1} u^{k}\right) u^{r} 0$. Since $b_{1}$ does not commute with $u, b_{1} u^{k}$ does not commute with $u$. By Lemma 5, there is a positive integer $n_{0}$ such that the first syllable of $u^{-n_{0}}\left(b_{1} u^{k}\right) u^{n_{0}}$ is in the same factor of $H$ as the first syllable of $u^{-1}$, the last syllable of $u^{-n_{0}}\left(b_{1} u^{k}\right) u^{n_{0}}$ is in the same factor of $H$ as the last syllable of $u$, the first syllable of $u^{n_{0}}\left(b_{1} u^{k}\right) u^{-n_{0}}$ is in the same factor of $H$ as the first syllable of $u$, and the last syllable of $u^{n_{0}}\left(b_{1} u^{k}\right) u^{-n_{0}}$ is in the same factor of $H$ as the last syllable of $u^{-1}$. Let $N_{1}$ be a normal subgroup of finite index in $G$ such that $h \not \equiv g^{z} \bmod N_{1}$ for all integers $z$ and all group elements $h$ ranging over the syllables of $u, a_{1}, u^{-y}\left(b_{1} u^{k}\right) u^{y}$ for $y>n_{0}$, and $b_{1}$ contained in the factor $G$ of $H$. $N_{1}$ exists since $G$ is $\Pi_{c}$. If $M$ is a normal subgroup of $G$ and a subgroup of $N_{1}$ and $K$ is the kernel of the naturally defined homomorphism from $H$ 
to $H^{*}=\left(x, G / M ; x^{m}=g^{*}\right)$, where $g^{*}$ is the image of $g$ in $G / M$, then

$$
a_{1}=u^{-r_{0}}\left(b_{1} u^{k}\right) u^{r_{0}} \bmod K
$$

is possible for only finitely many values of $r_{0}$. This is true because the homomorphism from $H$ to $H^{*}$ preserves the syllable lengths of $u, a_{1}, b_{1}$, and $u^{-y}\left(b_{1} u^{k}\right) u^{y}$ for $y>n_{0}$, and maps the syllables of these elements onto the syllables of their images in $H^{*}$. Thus the image of $u$ is cyclically reduced in $H^{*}$ and has syllable length greater than one. Thus for only finitely many integral $r_{0}$ can the syllable length of the image of $a_{1}$ equal the syllable length of the image of $u^{-r_{0}}\left(b_{1} u^{k}\right) u^{r_{0}}$, and these values of $r_{0}$ are independent of the way $M$ is chosen in $N_{1}$. Let $t_{1}, \ldots, t_{w}$ be the values of $r_{0}$ such that the syllable length of the image of $a_{1}$ in $H^{*}$ equals the syllable length of the image of $u^{-r_{0}}\left(b_{1} u^{k}\right) u^{r_{0}}$ in $H^{*}$.

Given a subscript $j$ between 1 and $w$, we consider the system of equations $\left(\mathrm{I}_{j}\right)$ as follows:

$$
\begin{aligned}
b_{1}^{-1} u^{t_{j}} a_{1} & =u^{r_{1}} \\
b_{2}^{-1} b_{1}^{-1} u^{t_{s}} a_{1} a_{2} & =u^{r_{2}} \\
& \vdots \\
b_{n-1}^{-1} \cdots b_{1}^{-1} u^{t_{j}} a_{1} \cdots a_{n-1} & =u^{r_{n-1}} \\
b_{n}^{-1} \cdots b_{1}^{-1} u^{t_{s}} a_{1} \cdots a_{n} & =u^{t_{j}} .
\end{aligned}
$$

If the system $\left(\mathrm{I}_{j}\right)$ has a solution $s_{1}, \ldots, s_{n-1}$, then the system of equations $a_{i}=$ $u^{-r_{i-1}} b_{i} u^{r_{i}}, r_{0}=r_{n}$, has the solution $r_{0}=t_{j}, r_{1}=s_{1}, \ldots, r_{n-1}=s_{n-1}$. Thus for each $j$ between 1 and $w$ the equations $\left(\mathrm{I}_{j}\right)$ have no solution. It follows from the remarks preceding Lemma 1 that $H$ is $\Pi_{c}$ and that there is a single normal subgroup $N_{2}$ of finite index in $H$ such that all the systems $\left(\mathrm{I}_{j}\right)$ for $j=1, \ldots, w$ have no solution modulo $N_{2}$. Let $M$ be a normal subgroup of $G$ contained in $G \cap N_{2}$, let $\psi_{1}$ be the natural homomorphism of $G$ onto $G / M$, and let $\varphi_{1}$ be the extension of $\psi_{1}$ to a homomorphism of $H$ onto $H^{*}=\left(x, G ; x^{m}=\psi_{1}(g)\right)$. Since $G \cap N_{2} \supset M$, there is a natural homomorphism $\psi_{2}$ of $G / M$ onto $G /\left(G \cap N_{2}\right)$, and $\psi_{2}$ can be extended to a homomorphism $\varphi_{2}$ of $H^{*}$ onto $H^{* *}=\left(x, G /\left(G \cap N_{2}\right) ; x^{m}=\psi_{2} \psi_{1}(g)\right)$. Since $\psi_{2} \psi_{1}$ is the restriction of the natural homomorphism of $H$ onto $H / N_{2}$ to $G$, there is a homomorphism $\varphi_{3}$ of $H^{* *}$ onto $H / N_{2}$. Since $N_{2}$ is the kernel of $\varphi_{3} \varphi_{2} \varphi_{1}, N_{2}$ contains the kernel of $\varphi_{1}$. Thus for $j=1, \ldots, w$, the equations $\left(\mathrm{I}_{j}\right)$ have no solution modulo the kernel of $\varphi_{1}$. Let $N_{3}=\left(G \cap N_{2}\right) \cap N_{3}, \psi$ be the natural homomorphism of $G$ onto $G / N_{3}$ and let $\varphi$ be the extension of $\psi$ to a homomorphism of $H$ onto $L=$ $\left(x, G / N_{3} ; x^{m}=\psi(g)\right)$. It follows from the results of this paragraph and the one preceding that the equations $\varphi\left(a_{i}\right)=\varphi(u)^{-r_{i-1}} \varphi\left(b_{i}\right) \varphi(u)^{r_{i}}, r_{0}=r_{n}$, have no solution with $r_{1}=r_{0}+k$. Note also that since $N_{1}$ and $G \cap N_{2}$ have finite index in $G, N_{3}$ has finite index in $G$.

Let $\eta$ be the natural homomorphism of $H$ onto $H / H^{\prime}$. Let $\delta$ be the homomorphism of $H$ onto $L \times H / H^{\prime}$ defined by $\delta(h)=\eta(h) \varphi(h)$ for all $h \in H$. Since $\eta\left(a_{i}\right)$ 


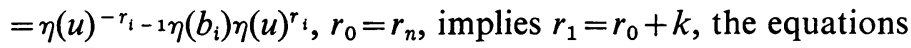

$$
\delta\left(a_{i}\right)=\delta(u)^{-r_{i}-1} \delta\left(b_{1}\right) \delta(u)^{r_{i}}, \quad r_{0}=r_{n},
$$

have no solution. Since $G / N_{3}$ is finite, it follows from theorems of H. Neumann and B. H. Neumann (cf. [8, Lemma 16]) that $L$ has a free subgroup of finite index. Since $H / H^{\prime}$ is free cyclic, it follows from Lemmas 2 and 3 that every element of $L \times H / H^{\prime}$ has property $\Pi$. Thus $\delta(u)$ has property $\Pi$ and there is a homomorphism $\xi$ of $L \times H / H^{\prime}$ onto a finite group such that the equations

$$
\xi \delta\left(a_{i}\right)=\xi \delta(u)^{-r_{i-1}} \xi \delta\left(b_{i}\right) \xi \delta(u)^{r_{i}}, \quad r_{0}=r_{n},
$$

have no solution. If $K$ is the kernel of $\xi \delta, K$ is of finite index in $H$. Thus the lemma is proven.

LeMmA 7. Let $G$ be $a \Pi_{c}$ group and let $g$ be an element of $G$ with property $\Pi$ in $G$. Let $m$ be an integer greater than one and let $H=\left(x, G ; x^{m}=g\right)$. If $h$ is a cyclically reduced element of syllable length greater than one in $H, h$ is c.d. in $H$.

Proof. Let $t$ be an element of $H$ not conjugate to $h$. We can assume that $t$ is cyclically reduced. Let $h=h_{1} \cdots h_{r}$ and $t=t_{1} \cdots t_{k}$, where the elements $h_{i}$ and $t_{i}$ are the syllables of $h$ and $t$ respectively. Since $G$ is $\Pi_{c}$, if $s$ ranges over the $h_{i}$ and $t_{i}$ in $G$, then there is a single normal subgroup $N_{1}$ of finite index in $G$ such that $s \not \equiv g^{z} \bmod N_{1}$ for all integers $z$ and all values of $s$. If $s$ is a syllable of $h$ in some representation of $h$ as a product of syllables, and $s$ is in $G$, there are integers $a, b$, and $c$ such that $s=g^{a} h_{b} g^{c}$ and $h_{b}$ is an element of $G$. Since $h_{b}$ is not congruent modulo $N_{1}$ to a power of $g, s$ is not congruent modulo $N_{1}$ to a power of $g$. Similarly, no syllable of $t$ in $G$ is congruent modulo $N_{1}$ to a power of $g$.

Suppose now that the syllable length of $t$ is not equal to the syllable length of $h$, i.e. $r \neq k$. Let $\psi$ be the natural homomorphism of $G$ onto $G / N_{1}$, and let $\varphi$ be the extension of $\psi$ to a homomorphism of $H$ onto $H^{*}=\left(x, G / N_{1} ; x^{m}=\psi(g)\right)$. The syllable length of $\varphi(h)$ is $r$, the syllable length of $\varphi(t)$ is $k$, and both $\varphi(h)$ and $\varphi(t)$ are cyclically reduced. Since $r \neq k, \varphi(h)$ is not conjugate to $\varphi(t)$. Since $\varphi(h)$ is cyclically reduced and has syllable length greater than one, it has infinite order. Since $G / N_{1}$ is finite, it follows as in the proof of Lemma 6 that $H^{*}$ has a free subgroup of finite index. According to [7, Theorem 2], $\varphi(h)$ is c.d. in $H^{*}$. Hence there is a homomorphism $\xi$ from $H^{*}$ onto a finite group such that $\xi \varphi(h)$ is not conjugate to $\xi \varphi(t)$.

Now suppose that $h$ and $t$ have the same syllable length, i.e. $r=k$. It follows from a theorem of D. Solitar [4, Theorem 4.6] that $h$ is conjugate to $t$ if and only if there is an integer $u_{0}$ and a cyclic permutation $\sigma$ of $i, \ldots, k$ such that $h=g^{-u_{0}} t_{\sigma(1)} \cdots t_{\sigma(n)} g^{u_{0}}$. Assume without loss of generality that the first syllable of $h$ is an element of $G$. Let $\sigma$ be a permutation of $1, \ldots, k$ such that $t_{\sigma(i)}$ is in the same factor of $H$ as $h_{i}$, and for $h_{i}$ in the factor of $H$ generated by $x, h_{i}=x^{u_{i}}, t_{\sigma(i)}=x^{v_{i}}$ with $u_{i}=v_{i} \bmod m$. If the above assumptions are true for $\sigma, t_{\sigma(1)} \cdots t_{\sigma(k)}=s_{1} h_{2} \cdots s_{k-1} h_{k}$, where $s_{i}=$ $t_{\sigma(i)} g^{\left(v_{i+1}-u_{i+1}\right) / m}$. Thus $h=g^{-r_{0} t_{\sigma(1)}} \cdots t_{\sigma(k)} g^{r_{0}}$ if and only if $h_{1} \cdots h_{k}=$ 
$g^{-r_{0}} s_{1} h_{2} \cdots s_{k-1} h_{k} g^{r_{0}}$. The latter equation holds if and only if there are integers $r_{0}, \ldots, r_{k-1}$ such that

$$
\begin{aligned}
h_{1} & =g^{-r_{0} S_{1}} g^{r_{1}} \\
h_{2} & =g^{-r_{1} h_{2} g^{r_{2}}} \\
& \vdots \\
h_{k-1} & =g^{-r_{k-2} S_{k-1} g^{r_{k-1}}} \\
h_{k} & =g^{-r_{k-1} h_{k} g^{r_{0}} .}
\end{aligned}
$$

The equations for $h_{2 n}$ imply that these equations have a solution if and only if there is a solution to the equations

$$
\begin{aligned}
h_{1} & =g^{-r_{0}} S_{1} g^{r_{2}} \\
h_{3} & =g^{-r_{2} S_{3}} g^{r_{4}} \\
& \vdots \\
h_{k-1} & =g^{-r_{k-2} S_{k-1}} g^{r_{0}} .
\end{aligned}
$$

Since $h$ is not conjugate to $t$, the latter system of equations has no solution. Since $h_{2 i+1}$ and $s_{2 i+1}$ are elements of $G$ and $g$ has property in $G$, there is a normal subgroup $N$ of finite index in $G$ such that the latter equations have no solution modulo $N$. Let $N$ be the intersection of $N_{1}$ and $N_{\sigma}$ for $\sigma$ such that $t_{\sigma(i)}$ is in the same factor of $H$ as $h_{i}$, and for $h_{i}$ in the factor of $H$ generated by $x, h_{i} \equiv t_{\sigma(i)} \bmod x^{m}$. Let $\eta$ be the natural homomorphism from $G$ onto $G / N$ and let $\psi$ be the extension of $\eta$ to a homomorphism from $H$ onto $H^{*}=\left(x, G / N ; x^{m}=\eta(g)\right)$. Clearly $N$ is a normal subgroup of finite index in $G$ so it follows as in the proof of Lemma 6 that $H^{*}$ has a free subgroup of finite index.

Suppose that $\psi(h)$ is conjugate to $\psi(t)$. Now $\psi(h)=\psi\left(h_{1}\right) \cdots \psi\left(h_{k}\right)$ and $\psi(t)=$ $\psi\left(t_{1}\right) \cdots \psi\left(t_{k}\right)$, where $\psi\left(h_{i}\right)$ and $\psi\left(t_{i}\right)$ are the syllables of $\psi(h)$ and $\psi(t)$ respectively. Since the theorem of D. Solitar used in the last paragraph applies to $H^{*}$, there is an integer $r_{0}$, and a cyclic permutation $\sigma$ of $1, \ldots, k$ such that

$$
\psi(h)=\psi(g)^{-r_{0}} \psi\left(t_{\sigma(1)}\right) \cdots \psi\left(t_{\sigma(k)}\right) \psi(g)^{r_{0}} .
$$

Thus there are integers $r_{1}, \ldots, r_{k-1}$ so that $\psi\left(h_{i}\right)=\psi(g)^{-r_{i-1}} \psi\left(t_{\sigma(i)}\right) \psi(g)^{r_{i}}, r_{n}=r_{0}$. These equations are soluble only if $\psi\left(h_{i}\right)$ and $\psi\left(t_{\sigma(i)}\right)$ are in the same factor of $H^{*}$, and if $\psi\left(h_{i}\right)$ is in the factor of $H^{*}$ generated by $x, \psi\left(t_{\sigma(i)}\right) \equiv\left(h_{i}\right) \bmod \psi(g)$. Since $N \subset N_{1}, h_{i}$ and $t_{\sigma(i)}$ must be in the same factor of $H$, and if $h_{i}$ and $t_{\sigma(i)}$ are in the factor of $H$ generated by $x, h_{i} \equiv t_{\sigma(i)} \bmod x^{m}$. Note that $x^{m}=g$. Thus $\sigma$ must be a permutation for which $N_{\sigma}$ is defined and $N_{\sigma} \supset N$. But $N_{\sigma}$ was chosen so that the relations between elements of $G$ implied by $h=g^{-r_{0}} t_{\sigma(1)} \cdots t_{\sigma(k)} g^{r_{0}}$ are not valid modulo $N_{\sigma}$. Thus $\psi(h)$ is not conjugate to $\psi(t)$ in $H^{*}$.

Since $H^{*}$ has a free normal subgroup of finite index, it follows from [7, Theorem 2] that elements of infinite order in $H^{*}$ are c.d. in $H^{*}$. Since $\psi(h)$ is cyclically reduced and has syllable length greater than one, $\psi(h)$ is c.d. in $H^{*}$. Since $\psi(t)$ is 
not conjugate to $\psi(h)$, there is a homomorphism $\xi$ of $H^{*}$ onto a finite group such that $\xi \psi(h)$ is not conjugate to $\xi \psi(t)$.

It now follows that the given element $h$ is c.d. in $H$.

LEMMA 8. Let $G$ be a $\Pi_{c} \cdots$ and c.s. group. Let $g$ be an element of $G$ with property $\Pi$ in $G$. Let $m$ be an integer greater than one. Let $H=\left(x, G ; x^{m}=g\right)$. If $h$ is an element of the subgroup $G$ of $H, h$ is c.d. in $H$.

Proof. Let $t$ be an element of $H$ not conjugate to $h$. Without loss of generality, we can assume that $t$ is cyclically reduced. If $t$ has syllable length greater than one, $t$ is c.d. in $H$ and there is a homomorphism $\xi$ of $H$ onto a finite group such that $\xi(t)$ and $\xi(h)$ are not conjugate. If $t$ has syllable length one and is not in $G, t$ is a power of $x$ not in $G$. Let $\eta$ be the homomorphism of $H$ onto a cyclic group of order $m$ generated by the image of $x$, where the kernel of $\eta$ is the normal closure of $G$ in $H$. Clearly, $\eta(t)$ is not conjugate to $\eta(h)$. Thus we can assume that $t$ is an an element of $G$. Since $G$ is c.s. and $h$ is not conjugate to $t$ in $G$, there is a normal subgroup $N$ of finite index in $G$ such that $u^{-1} t u \not \equiv h \bmod N$ for all $u$ in $G$. Let $\eta$ be the natural homomorphism of $G$ onto $G / N$. Let $P$ be the $m \times m$ permutation matrix corresponding to the cycle $(1 \cdots m)$. Let $H^{*}$ be the group of $m \times m$ matrices with entries in the integral group ring of $G / N$ with generators $\operatorname{diag}(a, \ldots, a)$ for all $a \in G / N$ and $\operatorname{diag}(1, \ldots, 1, \eta(g)) P$. The assignments $u \rightarrow \operatorname{diag}(\eta(u), \ldots, \eta(u))$, for $u \in G$ and $x \rightarrow \operatorname{diag}(1, \ldots, 1, \eta(g)) P$ clearly define a homomorphism $\psi$ of $H$ onto $H^{*}$. If $D$ is diagonal, $D P=P D^{*}$ where $D^{*}$ is diagonal. Thus it is clear that $H^{*}$ is finite and that if $\psi(h)$ and $\psi(t)$ are conjugate, then $\eta(h)$ and $\eta(t)$ are conjugate. Thus $\psi(h)$ is not conjugate to $\psi(t)$.

It now follows that $h$ is c.d. in $H$.

LEMMA 9. Let $G$ be $a \Pi_{c}$ and c.s. group. Let $g$ be an element of $G$ with property $\Pi$ in $G$. Let $m$ be an integer greater than one and let $H=\left(x, G ; x^{m}=g\right)$. If $H / H^{\prime}$ is a free cyclic group, if $g$ is not an element of $H^{\prime}$ and if $h$ is a power of $x$ not in $G$, then $h$ is c.d. in $H$.

Proof. Let $t$ be an element of $H$ not conjugate to $h$. Without loss of generality, we can assume that $t$ is cyclically reduced. If $t$ is an element of $G$ or has syllable length greater than one, $t$ is c.d. in $H$, so there is a homomorphism $\xi$ of $H$ onto a finite group such that $\xi(t)$ and $\xi(h)$ are not conjugate. Thus we can assume that $t$ is a power of $x$. Let $h=x^{n_{1}}, t=h^{n_{2}}$. Since $h$ is not conjugate to $t, n_{1} \neq n_{2}$. Let $\eta$ be the natural homomorphism of $H$ onto $H / H^{\prime}$. Since $\eta(g) \neq 1$ and $H / H^{\prime}$ is torsion free, $\eta(x) \neq 1$. Thus $\eta(h) \neq \eta(t)$. Let $\psi$ be a homomorphism of $H / H^{\prime}$ onto a finite group such that $\psi \eta(h) \neq \psi \eta(t)$. Since $\psi \eta(H)$ is abelian, $\psi \eta(h)$ is not conjugate to $\psi \eta(t)$.

It follows that the given element $h$ is c.d. in $H$.

LEMMA 10. Let $G$ be $a \Pi_{c}$ and c.s. group. Let $g$ be an element of $G$ with property $\Pi$ in $G$. Let $m$ be an integer with absolute value greater than one. Let $H=\left(x, G ; x^{m}=g\right)$. If $H / H^{\prime}$ is a free cyclic group, $H$ is c.s. 
Proof. First we show that $g$ is not an element of $H^{\prime}$. Suppose, to obtain a contradiction, that $g \in H^{\prime}$. Since there is a homomorphism of $H$ onto a cyclic group of order $m$ generated by the image of $x, x \notin H^{\prime}$. Since $x^{m}=g, g \in H^{\prime}$ implies that $H / H^{\prime}$ has torsion, contrary to hypothesis. Now $H$ is isomorphic to $\left(x, G ; x^{-m}=g\right)$ so that we may assume that $m$ is an integer greater than one. By Lemmas 7, 8 and 9, every cyclically reduced element of $H$ is c.d. in $H$. Since every element of $H$ is conjugate to a cyclically reduced element, $H$ is c.s.

THEOREM 1. The groups of hose knots are c.s.

Proof. Let $H$ be the group of a hose knot. We refer to the presentation given in the Introduction. Since $H$ is a knot group, $H / H^{\prime}$ is free cyclic. According to $\S 4$ of [6], the groups of hose knots are $\Pi_{c}$. According to Theorem 1 of [8], the groups of torus knots, i.e. $\left(a, b, a^{r}=b^{s}\right)$, are c.s. Thus it will follow that the groups of hose knots are c.s. if at each stage of the construction of the group by adding roots of elements, a root of a $\Pi$ element is added and the resultant group has free cyclic commutator quotient group. Since at each stage in the construction a knot group is obtained, at each stage we obtain a group with free cyclic commutator quotient group.

Let us examine a stage of the construction to see that a root of a $\Pi$ element is added. Let $H^{*}$ be the subgroup of the group of a hose knot generated by $P_{1}, Q_{1}, \ldots, Q_{i-1}$. We add $Q_{i}$ to $H^{*}$, where $Q_{i}$ is a root of the element

$$
g_{i}=P_{i}^{n_{i}-n_{i-1} m_{i}} Q_{i-\overline{1}^{1} n_{i}}^{n^{2}}
$$

It was shown in the proof of Lemma 10 that the resultant group has free cyclic commutator quotient group only if $g_{i}$ is not an element of the commutator subgroup of $H^{*}$. If we regard $H^{*}$ as the free product of its subgroups generated by $Q_{i-1}$ and $Q_{i-2}, \ldots, Q_{1}, P_{1}$ with a cyclic amalgamated subgroup, it is clear from the relations between the exponents in the presentation of $H^{*}$ and those of $g_{i}$ that $g_{i}$ is conjugate to a cyclically reduced element of length greater than one in $H^{*}$. Thus $g_{i}$ is, by Lemma 6 , conjugate to a $\Pi$ element of $H^{*}$ and hence is itself a $\Pi$ element of $H^{*}$. It thus follows inductively, using Lemmas 6 and 10, that the groups of hose knots are c.s.

\section{REFERENCES}

1. K. Brauner, Zur Geometrie der Funktionen Zweier komplexer Veraenderlicher, Abh. Math. Sem. Univ. Hamburg 6 (1928), 1-55.

2. M. Hall, Jr., A topology for free groups and related groups, Ann. of Math. (2) 52 (1950), 127-139. MR 12, 158.

3. S. Lipschutz, Generalization of Dehn's result on the conjugacy problem, Proc. Amer. Math. Soc. 17 (1966), 759-762. MR 33 \#5706.

4. W. Magnus, A. Karrass and D. Solitar, Combinatorial group theory, Wiley, New York, 1966. MR 34 \#7617.

5. A. W. Mostowski, On the decidability of some problems in special classes of groups, Fund. Math. 59 (1966), 123-135. MR 37 \#292. 
6. P. Stebe, Residual finiteness of a class of knot groups, Comm. Pure Appl. Math. 21 (1968), 563-583. MR 38 \#5902.

7. - A residual property of certain groups, Proc. Amer. Math. Soc. 26 (1970), 37-42.

8. - Conjugacy separability of certain free products with amalgamation, Trans. Amer. Math. Soc. 156 (1971), 119-129.

INSTITUTE FOR DEFENSE ANALYSES,

ARLINGTON, Virginia 22202 